\title{
UTILIZAÇÃO DOS EQUIPAMENTOS DE PROTEÇÃO INDIVIDUAL A PARTIR DA ÓTICA DA EQUIPE DE ENFERMAGEM NO ENFRENTAMENTO DO COVID-19
}

\section{USE OF PERSONAL PROTECTIVE EQUIPMENT FROM THE PERSPECTIVE OF THE NURSING TEAM IN THE FIGHT AGAINST COVID-19}

\section{USO DE EQUIPOS DE PROTECCIÓN PERSONAL DESDE EL PUNTO DE VISTA DEL EQUIPO DE ENFERMERÍA EN ENFRENTAMIENTO DEL COVID-19}

Júlio Gabriel Mendonça de Sousa1, Wanderson Alves Ribeiro², Amanda de Araujo Franco ${ }^{3}$, Amanda de Almeida Floriano ${ }^{4}$, Ana Beatriz Teodoro de Souza ${ }^{5}$, Bianca Lemos de Carvalho ${ }^{6}$, Jully Camara Guinancio ${ }^{7}$, Lilian Laine da Conceição Dias ${ }^{8}$

Submetido em: 20/04/2021

e24225 Aprovado em: 10/05/2021

\section{RESUMO}

Trata-se de uma revisão narrativa de literatura, ou seja, busca compreender de maneira ampla a literatura existente no meio científico com objetivo de refletir sobre as boas práticas de enfermagem na utilização dos equipamentos de proteção individual na prevenção ao COVID-19 no âmbito dos serviços de saúde. Os equipamentos de proteção individual (EPIs) são todos os dispositivos ou produtos de uso individual, utilizados pelo trabalhador, destinados à proteção de riscos suscetíveis de ameaça à segurança e à saúde no trabalho. A pandemia por COVID-19 representa um dos maiores desafios sanitários em escala mundial deste século. Situação epidemiológica da covid-19 no Brasil (20.04.2021) - Ministério da Saúde. O total de vidas perdidas para a covid-19 chegou a 378.003. Para dar conta da proposta do estudo, a amostra final foi composta apenas por 09 referências, sendo 07 artigos e 02 cartilhas de orientações. Por fim, a pandemia trouxe tempos inigualáveis e instáveis e com a velocidade rápida das mudanças de forma crescente, no entanto, informações confiáveis, atuais e de boa qualidade não são suficientes, faz-se necessário também pensar no papel da comunicação de forma adequada e eficaz de eliminar os riscos, ressaltar a importância da higienização das mãos e uso contínuo da máscara de forma correta.

PALAVRAS-CHAVE: Equipamentos de Proteção Individual. Enfermagem. Infecções por Coronavirus.

\footnotetext{
ABSTRACT

This is a narrative literature review, that is, it seeks to comprehensively understand the existing literature in the scientific community in order to reflect on good nursing practices in the use of personal protective equipment in the prevention of COVID-19 within health services. Personal protective equipment (PPE) is all devices or products for individual use, used by the worker, intended to protect risks that may threaten safety and health at work. The COVID-19 pandemic represents one of the greatest global health challenges of this century. Epidemiological situation of covid-19 in Brazil (20.04.2021) - Ministry of Health. The total number of lives lost to covid-19 reached 378,003. To account for the study proposal, the final sample consisted of only 09 references, with 07 articles and 02 guidebooks. Finally, the pandemic has brought unparalleled and unstable times and with the fast speed of rapid changes, however, reliable, current and good

1 Acadêmico da Graduação de Enfermagem da Universidade Federal do Rio de Janeiro. RJ, Brasil.

2 Enfermeiro. Mestre e Doutorando pelo programa acadêmico em ciências cuidado em saúde da EEAAC/UFF; Docente do curso de graduação em enfermagem da Universidade Iguaçu.

3 Enfermeira. Graduada pela Universidade Iguaçu, Nova Iguaçu, RJ, Brasil.

4 Enfermeira. Graduada pela Universidade Iguaçu, Nova Iguaçu, RJ, Brasil.

${ }^{5}$ Enfermeira. Graduada pela Universidade Iguaçu, Nova Iguaçu, RJ, Brasil.

${ }^{6}$ Enfermeira. Graduada pela Universidade Iguaçu, Nova Iguaçu, RJ, Brasil.

7 Enfermeira. Graduada pela Universidade Iguaçu, Nova Iguaçu, RJ, Brasil.

8 Enfermeira. Pós-Graduada em Enfermagem em Oncologia pela UCL.
} 


\section{RECIMA21 - REVISTA CIENTÍFICA MULTIDISCIPLINAR ISSN 2675-6218}

UTILIZAÇÃO DOS EQUIPAMENTOS DE PROTECÃO INDIVIDUAL A PARTIR DA ÓTICA DA EQUIPE DE ENFERMAGEM NO ENFRENTAMENTO DO COVID-19 DA EQUIPE DE ENFERMAGEM NO ENFRENTAMENTO DO COVID-19
Júlio Gabriel Mendonça de Sousa, Wanderson Alves Ribeiro, Amanda de Araujo Franco, Amanda de Almeida Floriano, Ana Beatriz Teodoro de Souza, Bianca Lemos de Carvalho, Jully Camara Guinancio, Lilian Laine da Conceição Dias

quality information is not enough, it is also necessary to think about the role of communication properly. It is effective in eliminating risks, emphasizing the importance of hand hygiene and continuous use of the mask correctly.

DESCRIPTORS: Personal Protective Equipment. Nursing. Coronavirus infections.

\section{RESUMEN}

Es una revisión narrativa de la literatura, es decir, busca comprender de manera integral la literatura existente en el medio científico con el fin de reflexionar sobre las buenas prácticas de enfermería en el uso de equipos de protección personal en la prevención de COVID-19 en el ámbito de la salud. servicios. Los equipos de protección personal (EPI) son todos los dispositivos o productos de uso individual, utilizados por el trabajador, destinados a proteger los riesgos que pueden amenazar la seguridad y la salud en el trabajo. La pandemia de COVID-19 representa uno de los mayores desafíos de salud mundial de este siglo. Situación epidemiológica del covid-19 en Brasil (20.04.2021) - Ministerio de Salud. El número total de vidas perdidas por covid-19 alcanzó las 378.003. Entre ayer y hoy se confirmaron 3.321 nuevas muertes. Para dar cuenta de la propuesta de estudio, la muestra final constó de solo 09 referencias, con 07 artículos y 02 guías. Por último, la pandemia ha traído tiempos inigualables e inestables y con la rapidez de los cambios vertiginosos, sin embargo, la información confiable, actual y de buena calidad no es suficiente, también es necesario pensar en el papel de la comunicación adecuadamente. Es eficaz en eliminar riesgos, destacando la importancia de la higiene de manos y el uso continuo de la mascarilla correctamente.

DESCRIPTORES: Equipo de protección personal. Enfermería. Infecciones por coronavirus.

\section{INTRODUÇÃO}

De acordo a Lei 6.514/1977, mais especificamente a Norma Regulamentadora (NR- 6), o EPI (Equipamento de Proteção Individual) é todo dispositivo de uso individual destinado a proteger a saúde e a integridade física do trabalhador, incluindo luvas, aventais, protetores oculares, faciais e auriculares, protetores respiratórios e para os membros inferiores. É responsabilidade do empregador o fornecimento do EPI adequado ao risco e o treinamento dos trabalhadores quanto à forma correta de utilização e conservação (BRASIL,2013; SUARTE et. al., 2013).

Seguindo tal premissa, podemos afirmar que estas atividades decorrentes do trabalho são, às vezes, responsáveis por danos físicos, em virtude da falta de conhecimento sobre medidas preventivas e do uso incorreto de equipamentos de proteção (TALHAFERRO; BARBOSA; FERREIRA, 2008).

Cabe ressaltar que a utilização dos equipamentos de proteção individual é de grande importância para os profissionais da equipe de enfermagem, uma vez que os mesmos estão expostos às doenças durante o manuseio das secreções dos pacientes e dos dejetos humanos, das roupas de cama sujas e de superfícies contaminadas, colocando os profissionais em risco mais elevado de contrair infecção e de se contaminar (DIAS et al., 2016). 


\section{RECIMA21 - REVISTA CIENTÍFICA MULTIDISCIPLINAR ISSN 2675-6218}

UTILIZAÇÃO DOS EQUIPAMENTOS DE PROTECÃO INDIVIDUAL A PARTIR DA ÓTICA DA EQUIPE DE ENFERMAGEM NO ENFRENTAMENTO DO COVID-19 DA EQUIPE DE ENFERMAGEM NO ENFRENTAMENTO DO COVID-19
Júlio Gabriel Mendonça de Sousa, Wanderson Alves Ribeiro, Amanda de Araujo Franco, Amanda de Almeida Floriano, Ana Beatriz Teodoro de Souza, Bianca Lemos de Carvalho, Jully Camara Guinancio, Lilian Laine da Conceição Dias

De acordo com Marziale et al., (2012), os EPI's que fazem parte da prática profissional de enfermagem são: máscaras para proteção respiratória; óculos para amparar os olhos contra impactos, radiações e substâncias; luvas para proteger contra riscos biológicos e físicos; avental ou capote descartável e gorro para evitar aspersão de partículas dos cabelos e do couro cabeludo (BARROS et al., 2016).

Corroborando ao contexto, cabe mencionar que a elevada infectividade de seu agente etiológico, aliada à ausência de imunidade prévia na população humana e à inexistência de vacina, contribui com que o crescimento do número de casos seja exponencial, se não forem tomadas medidas para deter sua transmissão (GARCIA, 2020).

No que se refere à relevância da utilização dos EPIs, de acordo com o cenário mundial, ainda que distante do cenário brasileiro, o início de 2020 foi marcado por um surto de uma misteriosa pneumonia causada por uma variação do coronavírus cujo primeiro caso foi reportado em dezembro de 2019 na cidade de Wuhan, na China (OLIVEIRA; LUCAS; IQUIAPAZA, 2020; OMS, 2020).

Trata-se de um vírus isolado pela primeira vez em 1937 e em 1965 descrito como coronavírus, em virtude de seu perfil na microscopia, semelhante a uma coroa. Entre 2002 e 2003, a OMS notificou 774 mortes devido à síndrome respiratória aguda grave, o coronavírus denominado SARS-CoV-2 e, em 2012, foram confirmadas 858 mortes causadas pela síndrome respiratória do oriente médio (Mers-CoV), na Arábia Saudita, ambas as complicações ocasionadas por membros da família do coronavírus (CHANH; YAN; WANG, 2020; OLIVEIRA; LUCAS; IQUIAPAZA, 2020).

Nesse sentido, a transmissão do Sars-CoV-2 de pessoa para pessoa se dá por meio da autoinoculação do vírus em membranas mucosas (nariz, olhos ou boca) e do contato com superfícies inanimadas contaminadas, o que tem chamado cada vez mais atenção para a necessidade de adoção rápida e preventiva de medidas de proteção humana a fim de impedir a contaminação de pessoas (OLIVEIRA; LUCAS; IQUIAPAZA, 2020).

Nessas circunstâncias são indicadas intervenções não farmacológicas, que incluem medidas com alcance individual, ambiental e comunitário, como a lavagem das mãos, a etiqueta respiratória, o distanciamento social, o arejamento e a exposição solar de ambientes, a limpeza de objetos e superfícies, e a restrição ou proibição ao funcionamento de escolas, universidades, locais de convívio comunitário, transporte público, além de outros locais onde há aglomeração de pessoas (GARCIA, 2020).

Acrescentam-se ainda que, as medidas preventivas incluem a higienização das mãos com água e sabão sempre que possível e uso de álcool em gel nas situações em que o acesso à água e ao sabão não fosse possível. Recomenda-se evitar tocar olhos, nariz e boca, e proteger as pessoas ao redor ao espirrar ou tossir, com adoção da etiqueta respiratória, pelo uso do cotovelo flexionado ou lenço descartável. Além disso, a OMS indicou a manutenção da distância social 


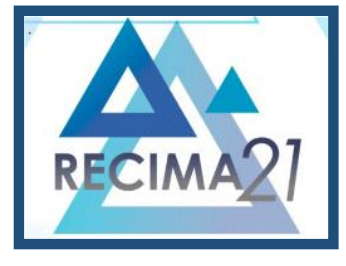

\section{RECIMA21 - REVISTA CIENTÍFICA MULTIDISCIPLINAR ISSN 2675-6218}

UTILIZAÇÃO DOS EQUIPAMENTOS DE PROTEC̃̃O INDIVIDUAL A PARTIR DA ÓTICA DA EQUIPE DE ENFERMAGEM NO ENFRENTAMENTO DO COVID-19 DA EQUIPE DE ENFERMAGEM NO ENFRENTAMENTO DO COVID-19
Júlio Gabriel Mendonça de Sousa, Wanderson Alves Ribeiro, Amanda de Araujo Franco, Amanda de Almeida Floriano, Ana Beatriz Teodoro de Souza, Bianca Lemos de Carvalho, Jully Camara Guinancio, Lilian Laine da Conceição Dias

(mínimo de um metro), que se evitassem aglomerações, e a utilização de máscara em caso de quadro gripal ou infecção pela COVID-19, ou se profissional de saúde no atendimento de pacientes suspeitos/infectados China (OLIVEIRA; LUCAS; IQUIAPAZA, 2020; OMS, 2020).

A pandemia por COVID-19 representa um dos maiores desafios sanitários em escala mundial deste século. Na primeira semana do mês de abril, poucos meses depois do início da epidemia na China, em dezembro de 2019, já foram reportados mais de 1,5 milhão de casos e 85 mil mortes no mundo, e espera-se que um número ainda maior de casos e óbitos venha a ocorrer nos próximos meses. No Brasil, até o dia oito de abril de 2020, foram registrados 15.927 casos confirmados e 800 mortes pelo COVID-19 (BARRETO et al., 2020).

Estas medidas, quando adotadas no início de um período epidêmico, auxiliam na prevenção da transmissão, na diminuição da velocidade de espalhamento da doença, e consequentemente contribuem para achatar a curva epidêmica. Assim, é possível diminuir a demanda instantânea por cuidados de saúde e mitigar as consequências da doença sobre a saúde das populações, incluindo a redução da morbidade e da mortalidade associadas (GARCIA, 2020).

Por sua vez, o distanciamento social também está entre as prioridades das instituições para diminuir a transmissão do Sars-CoV-2, minimizando o contato entre indivíduos potencialmente infectados e saudáveis, ou entre grupos com altas taxas de transmissão e ou aqueles com nenhum ou baixo nível, a fim de atrasar o pico da epidemia e diminuir a magnitude dos seus efeitos, para proteger a capacidade de assistência clínica (OLIVEIRA; LUCAS; IQUIAPAZA, 2020; OMS, 2020).

O insuficiente conhecimento científico sobre o novo coronavírus, sua alta velocidade de disseminação e capacidade de provocar mortes, geram incertezas quanto à escolha das melhores estratégias a serem utilizadas para o enfrentamento da epidemia em diferentes partes do mundo, No Brasil, os desafios que se apresentam são ainda maiores, pois pouco se sabe sobre as características de transmissão da COVID-19 num contexto de grande desigualdade social e demográfica, com populações vivendo em condições precárias de habitação e saneamento, sem acesso constante à água, em situação de aglomeração e com alta prevalência de doenças crônicas (GARCIA, 2020).

Um contexto de pandemia exige mudanças significativas e frequentes nos hábitos de vida e nas rotinas dos profissionais, nos protocolos e fluxos institucionais, o que requer que os profissionais compreendam este momento de crise como oportunidade para elevar a profissão a patamares sociais nunca antes vistos em nosso país e que esse reconhecimento social possa mudar práticas e políticas referentes à enfermagem (OLIVEIRA; LUCAS; IQUIAPAZA, 2020).

$O$ cotidiano de atuação requer que o profissional revisite suas competências, habilidades e atitudes e as adeque ao momento atual para o desenvolvimento do cuidado, compreendendo que esse cuidado se desenvolve em níveis de complexidade distintos, porém indissociáveis, que 


\section{RECIMA21 - REVISTA CIENTÍFICA MULTIDISCIPLINAR ISSN 2675-6218}

UTILIZAÇÃO DOS EQUIPAMENTOS DE PROTEC̃̃O INDIVIDUAL A PARTIR DA ÓTICA DA EQUIPE DE ENFERMAGEM NO ENFRENTAMENTO DO COVID-19 DA EQUIPE DE ENFERMAGEM NO ENFRENTAMENTO DO COVID-19
Júlio Gabriel Mendonça de Sousa, Wanderson Alves Ribeiro, Amanda de Araujo Franco, Amanda de Almeida Floriano, Ana Beatriz Teodoro de Souza, Bianca Lemos de Carvalho, Jully Camara Guinancio, Lilian Laine da Conceição Dias

transitam desde uma simples ação educativa sobre lavagem das mãos, utilização dos EPIs e até as práticas de cuidado na alta complexidade (RAMOS, 2020).

Os hospitais estão sobrecarregados com o atendimento dos pacientes infectados pelo vírus, portanto, é salutar que as instituições destinadas ao atendimento de pacientes oncológicos sejam mantidas o maior tempo possível livre de pacientes com diagnóstico firmado para a Covid19. Nesse contexto, a enfermagem brasileira está enfrentando grandes desafios, sem precedentes históricos em sua atuação, nos diversos cenários da assistência à saúde, sendo convocada a revisitar a sua prática e a elaborar novas estratégias para minimizar os impactos gerados pela pandemia no atendimento aos pacientes com COVID-19 (CHANH; YAN; WANG, 2020).

Diante da temática exposta, emerge a seguinte questão norteadora: Como a equipe de enfermagem tem utilizado equipamentos de proteção individual na prevenção ao COVID-19 no âmbito hospitalar?

Nesse sentido, o artigo tem como objetivo refletir sobre as boas práticas da equipe de enfermagem na utilização dos equipamentos de proteção individual na prevenção ao COVID-19 no âmbito hospitalar.

\section{METODOLOGIA}

Trata-se de uma revisão narrativa de literatura, ou seja, busca compreender de maneira ampla a literatura existente no meio científico, favorecendo observar prováveis "deficiências" acerca do conhecimento de determinado assunto (BRUM et al., 2015).

A construção do artigo foi realizada a partir de pesquisa bibliográfica que, segundo Gil (2008) é desenvolvida com auxílio de material já elaborado, constituído principalmente de livros e artigos científicos. Contudo, mesmo que em grande parte dos estudos seja exigido algum tipo de trabalho deste gênero, há pesquisas desenvolvidas exclusivamente a partir de fontes bibliográficas.

O estudo realizou um levantamento bibliográfico dos últimos 10 anos nas bases de dados, Banco de Dados de Enfermagem (BDENF), Literatura Latino-Americana e do Caribe em Ciências da Saúde (LILACS), Medical Literature Analysis and Retrieval Sistem On-line (Medline) e Google Scholar. O levantamento dos dados ocorreu no mês de maio de 2020 por meio dos seguintes descritores: "Equipamentos de Proteção Individual"; "Enfermagem"; "Infecções por Coronavirus".

Como critérios de inclusão foram utilizados: ser artigo científico, estar disponível on-line, em português, na íntegra gratuitamente e versar sobre a temática pesquisada e ainda, revisões e cartilhas de orientação. Cabe mencionar que os textos em língua estrangeira foram excluídos devido o interesse em embasar o estudo com dados do panorama brasileiro e os textos incompletos, para oferecer melhor compreensão através da leitura de textos na íntegra. 


\section{RECIMA21 - REVISTA CIENTÍFICA MULTIDISCIPLINAR ISSN 2675-6218}

UTILIZAÇÃO DOS EQUIPAMENTOS DE PROTEC̃̃O INDIVIDUAL A PARTIR DA ÓTICA DA EQUIPE DE ENFERMAGEM NO ENFRENTAMENTO DO COVID-19 Júlio Gabriel Mendonça de Sousa, Wanderson Alves Ribeiro, Amanda de Araujo Franco, Amanda de Almeida Floriano, Ana Beatriz Teodoro de Souza, Bianca Lemos de Carvalho, Jully Camara Guinancio, Lilian Laine da Conceição Dias

Porém, para que atendessem os critérios de exclusão das referências supracitadas, primeiramente, foi realizada a leitura do título e/ou resumo, no qual foram desconsideradas todas as produções duplicadas. Vale informar que, o processo de revisão das referências foi desenvolvido por todos os autores, permitindo desta forma um melhor processamento e escolha das referências a serem utilizadas na edificação da presente pesquisa.

A amostra final foi composta apenas por 54 referências, sendo 28 artigos e 02 cartilhas de orientações, incluindo apenas as de maior relevância para este estudo, que apresentassem um embasamento científico que contribuíssem significativamente ao desenvolvimento deste artigo.

Figura 1 - Fluxograma das referências selecionadas.

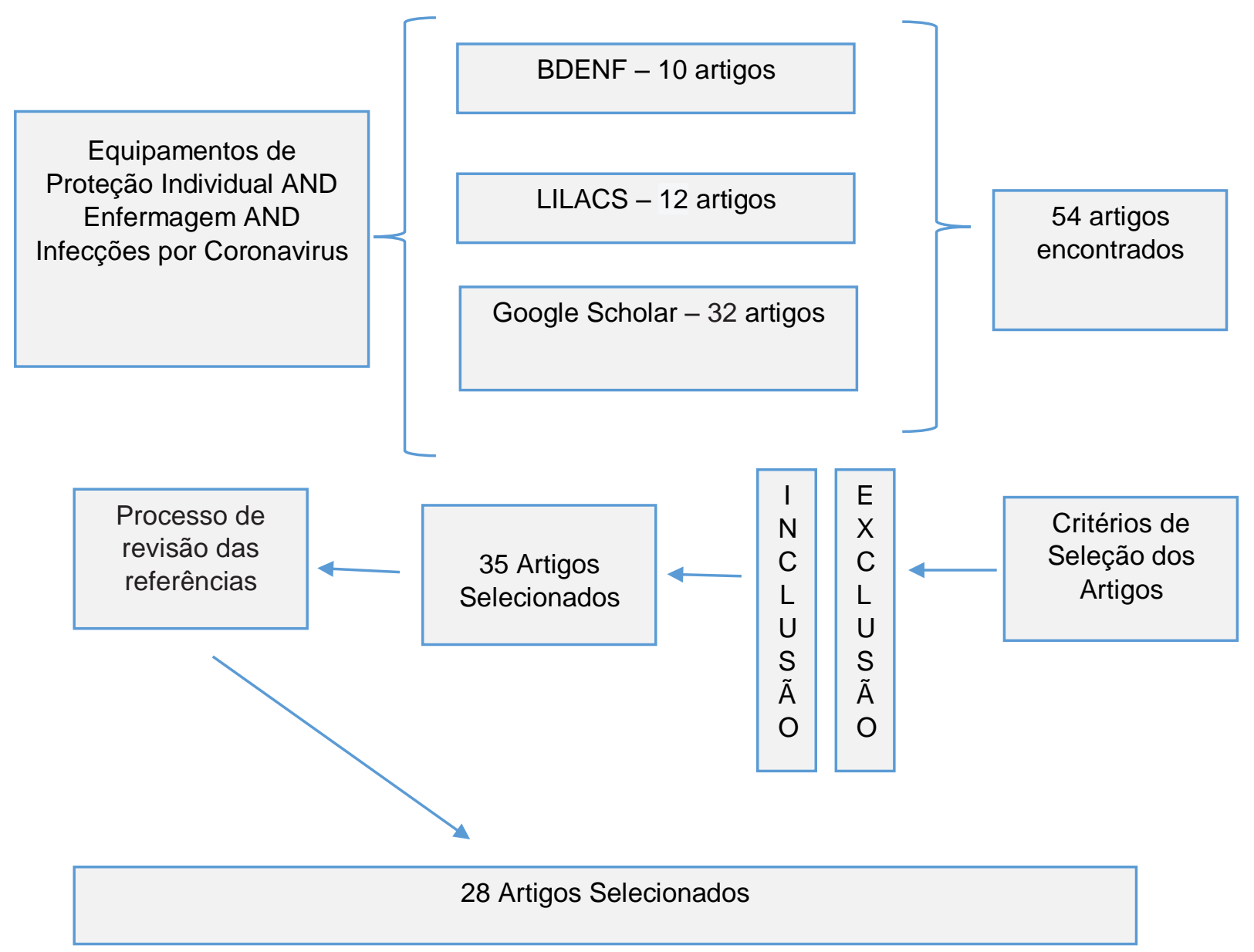

Fonte: Produção dos autores, 2021.

Após a leitura reflexiva dos artigos selecionados, foi possível discutir os resultados, categorizando os achados. 


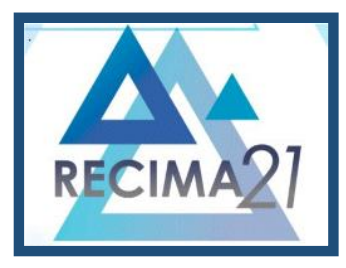

RECIMA21 - REVISTA CIENTÍFICA MULTIDISCIPLINAR ISSN 2675-6218

UTILIZAÇÃO DOS EQUIPAMENTOS DE PROTEC̄̃O INDIVIDUAL A PARTIR DA ÓTICA DA EQUIPE DE ENFERMAGEM NO ENFRENTAMENTO DO COVID-19 DA EQUIPE DE ENFERMAGEM NO ENFRENTAMENTO DO COVID-19
Júlio Gabriel Mendonça de Sousa, Wanderson Alves Ribeiro, Amanda de Araujo Franco, Amanda de Almeida Floriano, Ana Beatriz Teodoro de Souza, Bianca Lemos de Carvalho, Jully Camara Guinancio, Lilian Laine da Conceição Dias

\section{RESULTADO E DISCUSSÃO}

\section{Qualidade e efetividade de proteção dos EPI's dentro de uma unidade de saúde.}

As boas práticas incluem higienização das mãos, manipulação e descarte de material perfuro cortante de maneira segura e preventiva, uso de equipamentos de proteção individual de modo a proteger os profissionais, os pacientes e o meio ambiente e a imunização dos trabalhadores e tais recomendações devem ser adotadas no atendimento a todo e qualquer paciente independente de seu diagnóstico (PORTO; MARIZALE, 2016).

Posto que, uma das medidas mais importantes para a prevenção da transmissão se refere à higiene das mãos, considerada uma medida de baixo custo e alta efetividade, por serem as mãos o principal veículo de contaminação cruzada. Embora inúmeros trabalhos apontem para a inadequada adoção dessa prática entre os profissionais durante o atendimento de pacientes nos serviços de saúde. Reconhecidamente, a prática da higienização das mãos pela ação de fricção com água e sabão diminui a ocorrência das infecções previsíveis, reduzindo a morbimortalidade em serviços de saúde (OLIVEIRA; LUCAS; IQUIAPAZA, 2020).

Em relação aos fatores de risco associados à infecção, ressalta-se o fato que para as medidas de proteção do profissional de enfermagem, o uso de EPIs é fundamental. Destaca-se a importância de treinamentos no manuseio adequado desses equipamentos, como, por exemplo, os processos de colocação e retirada dos EPIs e treinamento presencial do uso de EPI. Entretanto, a rotina e os hábitos que propiciam potencializar a contaminação entre os PS também são altamente relevantes. Nesse sentido, deve-se propor uma estratégia com o intuito de modificá-las, conscientizando os PS que a transmissão pode acontecer inclusive durante as refeições e reuniões em grupo (SANT'ANA, et al., 2020).

Diante do exposto, ressalta-se que as estratégias para lidar com a presente situação de pandemia podem citar o fornecimento de EPIs, o treinamento adequado e o reforço no hábito do uso de EPI, a proteção dos olhos e a adoção de precaução padrão. Além disso, devem ser instituídas medidas para controle da transmissão de modo universal no ambiente hospitalar, mesmo quando esteja em contato apenas com pacientes sem suspeita de COVID-19 ou com outros trabalhadores (SANT'ANA, et al., 2020).

Corroborando ao contexto, ressalta-se que os profissionais da equipe de enfermagem tornam-se vulneráveis em função de algumas características que lhe são próprias, tais como: eles são o maior grupo individual da saúde prestador de assistência ininterrupta, 24 horas por dia, executam cerca de $60 \%$ das ações de saúde, realizam o maior volume de cuidado direto por meio de contato físico com o doente e executam rotineiramente procedimentos invasivos, principalmente administração de medicação injetável, o que predispõe à ocorrência de infecção cruzada (SANTOS et al., 2012), que se torna uma grande preocupação para o cenário atual da 


\section{RECIMA21 - REVISTA CIENTÍFICA MULTIDISCIPLINAR ISSN 2675-6218}

UTILIZAÇÃO DOS EQUIPAMENTOS DE PROTECÃO INDIVIDUAL A PARTIR DA ÓTICA DA EQUIPE DE ENFERMAGEM NO ENFRENTAMENTO DO COVID-19 DA EQUIPE DE ENFERMAGEM NO ENFRENTAMENTO DO COVID-19
Júlio Gabriel Mendonça de Sousa, Wanderson Alves Ribeiro, Amanda de Araujo Franco, Amanda de Almeida Floriano, Ana Beatriz Teodoro de Souza, Bianca Lemos de Carvalho, Jully Camara Guinancio, Lilian Laine da Conceição Dias

pandemia da COVID-19, onde o profissional de saúde pode contribuir, sendo um veículo de transmissão de um paciente para o outro.

Estudos que avaliaram a eficácia do isolamento de casos no controle da COVID-19 mostraram que o isolamento pode ser suficiente para seu controle em um período de três meses, tendo sido mais eficaz quando havia baixa transmissão, antes do início dos sintomas. No entanto, apenas $\mathrm{o}$ isolamento, sem as medidas adequadas de precaução pode ser considerado insuficiente para controlar o surto. Assim, a sociedade/comunidade, neste momento, é alertada para a importância da técnica correta de higiene das mãos, uso de máscara e medidas de higiene de superfície que evitam em conjunto à disseminação do vírus (OLIVEIRA; LUCAS; IQUIAPAZA, 2020; COREN, 2020).

Cabe ressaltar que a COVID-19 pode ser transmitida por contato direto ou indireto (superfícies com resquício de gotículas/ aerossóis) ou transmissão de gotículas, sendo esta atribuída às partículas respiratórias de tamanho considerado grande, estando elas sujeitas às forças gravitacionais. Assim, as gotículas viajam pelo ar - considerado o indivíduo doente parado apenas a aproximadamente um metro do paciente, enquanto existem estudos provando que em movimento (como exemplo, durante uma corrida), estas gotículas podem chegar a dez metros do indivíduo infectado (COOK, 2020).

Contribui-se ainda que o uso de EPI deva considerar o nível de cuidado e tipo de atividade a ser executada: triagem, amostra para diagnóstico laboratorial, caso suspeito ou confirmado de 2019-nCov que requer internação na unidade de saúde e sem procedimento gerador de aerossol (PGA) e, por fim, caso suspeito ou confirmado de 2019-nCov que requer internação na unidade de saúde e PGA. Recomenda-se adotar precauções-padrão, de contato e de transmissão por gotículas a todos os casos suspeitos ou confirmados de Covid-19 e, em situações específicas, utilizar precauções por aerossol (SOARES et al., 2020).

No que se refere ao uso da máscara cirúrgica, cabe mencionar que para pacientes reduz a transmissão do aerossol, quando nos casos de contato com pessoas suspeitas de COVID-19 e com sintomas respiratórios brandos, desde a chegada ao serviço de saúde, ao local de isolamento e durante a circulação dentro do serviço (transporte de uma área/setor para outro) devendo-se evitar ao máximo tocar a máscara, olhos, boca e face (OLIVEIRA; LUCAS; IQUIAPAZA, 2020).

Essa medida pode limitar a propagação de doenças respiratórias, incluindo o novo coronavírus. No entanto, apenas o uso da máscara é insuficiente para fornecer o nível seguro de proteção isoladamente, devendo ser sempre associada àquelas já referidas como a higiene das mãos, sobretudo antes e após a utilização das máscaras. Deve-se lembrar de ainda que usar máscaras quando não indicado pode gerar custos desnecessários e criar uma falsa sensação de segurança, induzindo a negligência a outras medidas, como a prática de higiene das mãos e a higienização de superfícies inanimadas potencialmente contaminadas com o Sars-CoV-2 (OLIVEIRA; LUCAS; IQUIAPAZA, 2020; COREN, 2020). 


\section{RECIMA21 - REVISTA CIENTÍFICA MULTIDISCIPLINAR ISSN 2675-6218}

UTILIZAÇÃO DOS EQUIPAMENTOS DE PROTEÇ̃̃ INDIVIDUAL A PARTIR DA ÓTICA DA EQUIPE DE ENFERMAGEM NO ENFRENTAMENTO DO COVID-19 DA EQUIPE DE ENFERMAGEM NO ENFRENTAMENTO DO COVID-19
Júlio Gabriel Mendonça de Sousa, Wanderson Alves Ribeiro, Amanda de Araujo Franco, Amanda de Almeida Floriano, Ana Beatriz Teodoro de Souza, Bianca Lemos de Carvalho, Jully Camara Guinancio, Lilian Laine da Conceição Dias

As máscaras cirúrgicas devem ter alta resistência ao fluido; boa respirabilidade; no mínimo, uma camada interna e externa; e, obrigatoriamente, um elemento filtrante. Devem ser confeccionadas de forma a cobrir adequadamente a área do nariz e da boca do usuário, possuírem clipes nasais constituídos de material maleável que permitam ajuste adequado do contorno do nariz e das bochechas. Tais máscaras não devem ser sobrepostas à máscara N95 ou equivalente, pois além de não garantirem proteção de filtração ou de contaminação, contribui com o desperdício de mais um EPI (SOARES et AL., 2020).

Os demais componentes de precaução e proteção profissional devem estar alinhados ao tipo de contato e procedimento a ser realizado e à paramentação para assistência ao paciente suspeito/portador da COVID-19 e incluem o uso de luvas, capote/avental, óculos de proteção ou protetor facial, gorro e avental (OLIVEIRA; LUCAS; IQUIAPAZA, 2020).

Complementa-se ainda que, há que se manter a atenção para as demais medidas preventivas já recomendadas, como distanciamento social e manutenção das mãos longe dos olhos, nariz e boca, além da higienização das mãos de forma adequada. Essa indicação se justifica pelo fato de que a máscara de tecido pode reduzir a disseminação do vírus por pessoas assintomáticas ou pré-sintomáticas que podem estar transmitindo o vírus sem saber, porém, não protege o indivíduo que a está utilizando, por não possuir capacidade de filtragem de microrganismos. Destaca-se que seu uso deve ser individual, não podendo ser compartilhado, e que, em serviços de saúde, máscaras de tecido não devem ser usadas sob qualquer circunstância, considerando o disposto na Nota técnica no 4/2020, da Agência Nacional de Vigilância Sanitária (ANVISA, 2020).

De acordo com os estudos de Junior et al., (2020) e Carvalho et al., (2020) pacientes com sintomas respiratórios, protocolos de triagem em pronto-atendimento devem ser criados, guiando de forma responsável pacientes potencialmente suspeitos para isolamento rápido e seguro, e orientação adequada quanto ao uso de máscaras para todos. Sabe-se que o uso correto dos EPIs é um importante meio de contenção da COVID-19, no entanto, deve-se associar, para um melhor resultado, orientações básicas de higiene pessoal.

Diante disso, informações adequadas sobre a lavagem das mãos, uso frequente do álcool em gel, maior cuidado pelo contato direto com secreções respiratórias e evitar a manipulação de áreas como olhos, nariz e boca ao espirrar e tossir podem auxiliar o combate a atual pandemia. Evitar o contato próximo com pessoas sintomáticas, atitudes como utilizar a parte interna do cotovelo ou tecido quando tossir ou espirrar são orientações pertinentes como medidas complementares (JUNIOR et al., 2020; CARVALHO et al., 2020).

\section{Tipos de EPIs e as recomendações para o uso de forma correta:}




\section{RECIMA21 - REVISTA CIENTÍFICA MULTIDISCIPLINAR ISSN 2675-6218}

UTILIZAÇÃO DOS EQUIPAMENTOS DE PROTEC̃̃O INDIVIDUAL A PARTIR DA ÓTICA DA EQUIPE DE ENFERMAGEM NO ENFRENTAMENTO DO COVID-19 DA EQUIPE DE ENFERMAGEM NO ENFRENTAMENTO DO COVID-19
Júlio Gabriel Mendonça de Sousa, Wanderson Alves Ribeiro, Amanda de Araujo Franco, Amanda de Almeida Floriano, Ana Beatriz Teodoro de Souza, Bianca Lemos de Carvalho, Jully Camara Guinancio, Lilian Laine da Conceição Dias

Quanto às máscaras, é importante estabelecer um padrão inicial para uso. Primeiramente, seja para uso profissional, ou para uso da população geral, as máscaras de proteção devem estar com sua estrutura completamente intacta, sem umidade, e/ou sujeira (AĞALAR; ENGIN; ÖZTÜRK, 2020; CARVALHO et al., 2020).

Podemos relacionar os tipos de EPIs com o seu uso adequado em cada situação de acordo com o Quadro 1.

Quadro 1. Tipos de EPIs de acordo com a situação de uso. Rio de Janeiro, RJ, Brasil, 2021

\begin{tabular}{|c|c|c|}
\hline $\begin{array}{l}\text { MODO DE } \\
\text { TRANSMISSÃO }\end{array}$ & $\begin{array}{l}\text { QUANDO USAR EM PACIENTE } \\
\text { COM COVID-19 }\end{array}$ & EPI \\
\hline $\begin{array}{c}\text { Precaução de } \\
\text { contato }\end{array}$ & $>2 \mathrm{~m}$ do paciente & Luvas, avental. \\
\hline $\begin{array}{l}\text { Precaução de } \\
\text { gotículas }\end{array}$ & $<2$ m do paciente & $\begin{array}{l}\text { Luvas, avental, máscara } \\
\text { cirúrgica resistente a fluidos, óculos } \\
\text { de proteção*. }\end{array}$ \\
\hline $\begin{array}{l}\text { Precaução de } \\
\text { aerossóis }\end{array}$ & $\begin{array}{c}\text { Procedimentos que gerem } \\
\text { aerossóis }\end{array}$ & $\begin{array}{c}\text { Luvas, avental de mangas compridas } \\
\text { repelente a } \\
\text { líquidos, óculos de proteção*, } \\
\text { máscara EFP3. }\end{array}$ \\
\hline
\end{tabular}

Nota: *Óculos de proteção podem ser substituídos por face shield (CARVALHO et al., 2020).

Fonte: Adaptado da Association of Anaesthetists (COOK, 2020; CARVALHO et al., 2020, p.3).

No Quadro 2, podemos visualizar a relação entre as recomendações para cada tipo de EPIs.

Quadro 2. Tipos de máscaras e recomendações. Rio de Janeiro, RJ, Brasil, 2021

\begin{tabular}{|c|c|c|}
\hline TIPO DE MÁSCARA & PREVENÇÃO & QUANDO USAR \\
\hline Máscara facial cirúrgico tipo IIR & $\begin{array}{l}\text { Gotículas em até } 1- \\
2 m\end{array}$ & $\begin{array}{l}\text { Proteção contra } \\
\text { gotículas transmissão }\end{array}$ \\
\hline 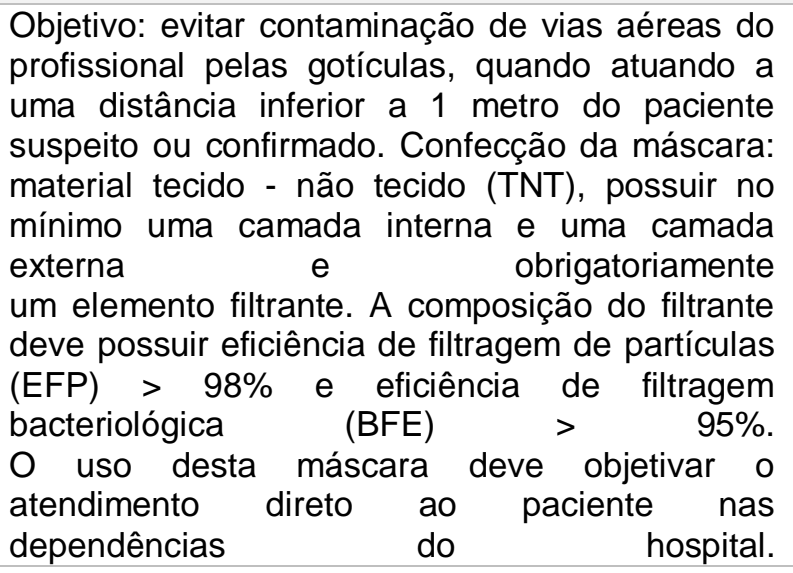 & & \\
\hline
\end{tabular}




\section{RECIMA21 - REVISTA CIENTÍFICA MULTIDISCIPLINAR ISSN 2675-6218}

UTILIZAÇÃO DOS EQUIPAMENTOS DE PROTEC̄̃O INDIVIDUAL A PARTIR DA ÓTICA DA EQUIPE DE ENFERMAGEM NO ENFRENTAMENTO DO COVID-19
Júlio Gabriel Mendonça de Sousa, Wanderson Alves Ribeiro, Amanda de Araujo Franco, Amanda de Almeida Floriano, Ana Beatriz Teodoro de Souza, Bianca Lemos de Carvalho, Jully Camara Guinancio, Lilian Laine da Conceição Dias

Devem utilizar essa máscara os pacientes com sintomas de infecção respiratória (febre, tosse, espirros, dispnéia).

Máscara de Proteção Respiratória (Respirador Particulado - N95 ou Equivalente)

Objetivo: proteger o profissional da saúde em procedimentos com risco de geração de aerossóis, como intubação ou aspiração traqueal, ventilação não invasiva, ressuscitação cardiopulmonar, ventilação manual antes da intubação, coletas de nasotraqueais e broncoscopias secreções. Deve ter eficácia mínima na filtração de $95 \%$ de partículas de até 0,3 $\mu$ (tipo N95, N99, N100, PFF2 ou PFF3).

$\mathrm{Na}$ rotina hospitalar, considera-se o uso de respiradores ou máscaras N95 ou equivalente, além do prazo de validade dado pelo fabricante para atendimento emergencial aos casos suspeitos ou confirmados da COVID-19. No entanto, as máscaras além do prazo de validade podem não possuir a mesma eficácia e não cumprir os requisitos para os quais foram certificados. Com o tempo, componentes como as tiras e o material da ponte nasal podem se degradar, o que pode afetar a qualidade do ajuste $\quad$ e da vedação. A máscara cirúrgica não deve se sobrepor à máscara N95 ou equivalente, pois além de não garantir proteção de filtração ou de contaminação, pode levar ao desperdício de mais um EPI, o que pode ser muito prejudicial em um cenário de escassez. Para reduzir a contaminação da máscara N95 ou equivalente, se houver disponibilidade, pode ser usado 0 face shield. Caso a máscara estiver íntegra, sem sujidade ou umidade, poderá ser utilizada várias vezes durante 0 mesmo plantão pelo mesmo profissional (até 12 horas ou conforme definido pela Comissão de Controle de Infecção Hospitalar - CCIH do serviço de saúde). Essa recomendação poderá ser atualizada. Para remover a máscara com o intuito de utilizá-la, retire-a pelos elásticos com extremo cuidado para não tocar a superfície interna e acondicione em um saco ou envelope de papel com os elásticos para fora, para facilitar a retirada da máscara. Nunca coloque a máscara para reuso em um saco plástico, pois ela poderá ficar úmida e potencialmente contaminada.

Máscara de Tecido
Aerossol

Proteção em

procedimentos com risco de

geração de aerossol 


\section{RECIMA21 - REVISTA CIENTÍFICA MULTIDISCIPLINAR ISSN 2675-6218}

UTILIZAÇÃO DOS EQUIPAMENTOS DE PROTEC̃̃O INDIVIDUAL A PARTIR DA ÓTICA DA EQUIPE DE ENFERMAGEM NO ENFRENTAMENTO DO COVID-19
Júlio Gabriel Mendonça de Sousa, Wanderson Alves Ribeiro, Amanda de Araujo Franco, Amanda de Almeida Floriano, Ana Beatriz Teodoro de Souza, Bianca Lemos de Carvalho, Jully Camara Guinancio, Lilian Laine da Conceição Dias

Serve como uma barreira mecânica contra disseminação do vírus por pessoas assintomáticas ou pré-sintomáticas que podem estar transmitindo o vírus sem conhecimento. Essa máscara não protege o indivíduo que a está utilizando, por não possuir capacidade de filtragem. Deve ser lavada, seca e por fim, passála em ferro quente fará uma limpeza mais profunda. O uso da máscara de tecido deve ser individual, não devendo ser compartilhado. Em serviços de saúde, sua utilização não é recomendada. Destaca-se aqui a importância do distanciamento social, evitar tocar os olhos, nariz e boca, além de higienizar as mãos com água e sabonete ou álcool gel a $70 \%$.

Nota: *Eficiência de Filtragem de Partículas: EFP2, EFP3 e N95: referem-se do desempenho de filtração das máscaras. Essa filtração é feita através da união de uma teia de polipropileno (microfibras) e carga eletrostática. Para FFP1, FFP2 e FFP3 a redução da concentração da substância é de 4, 10 e 20 vezes, respectivamente. ${ }^{* *}$ N95: em condições de teste, o respirador bloqueia ao menos $95 \%$ dos sólidos e líquidos das partículas aerossóis.

Fonte: CARVALHO et al., (2020, p.4).

De acordo com o Quadro 3, vê-se a relação de outros tipos de EPIs e suas recomendações.

Quadro 3. Outros tipos de EPIs e suas recomendações. Rio de Janeiro, RJ, Brasil, 2021

Luvas

Devem ser utilizadas, no contexto da epidemia da COVID-19, em qualquer contato com o paciente ou seu entorno (Precaução de Contato). As luvas devem ser colocadas antes da entrada no quarto do paciente ou área em que o paciente está isolado. Jamais sair do quarto ou área de isolamento com as luvas.

Protetor Ocular ou Devem ser utilizados quando houver risco de exposição do profissional Protetor de Face a respingos de sangue, secreções corporais e excreções.

(Face Shield)

Capote/Avental $\quad$ A necessidade do uso de capote ou avental impermeável (estrutura impermeável e gramatura mínima de $50 \mathrm{~g} / \mathrm{m} 2$ ) depende do quadro clínico do paciente (vômitos, diarreia, hipersecreção orotraqueal, sangramento etc.).

Gorro O gorro está indicado para a proteção dos cabelos e cabeça dos profissionais em procedimentos que podem gerar aerossóis.

Fonte: CARVALHO et al., (2020, p.4). 
UTILIZAÇÃO DOS EQUIPAMENTOS DE PROTEC̃̃O INDIVIDUAL A PARTIR DA ÓTICA DA EQUIPE DE ENFERMAGEM NO ENFRENTAMENTO DO COVID-19
Júlio Gabriel Mendonça de Sousa, Wanderson Alves Ribeiro, Amanda de Araujo Franco, Amanda de Almeida Floriano, Ana Beatriz Teodoro de Souza, Bianca Lemos de Carvalho, Jully Camara Guinancio, Lilian Laine da Conceição Dias

\section{O uso correto da paramentação como forma de prevenção da COVID-19:}

Em nota técnica publicada pela ANVISA, na qual a agência propõe que profissionais da saúde que realizem procedimentos a menos de um metro de distância, quais podem ser incorporadas às rotinas dos indivíduos, no que se refere à higiene pessoal de pacientes suspeitos ou confirmados para a infecção de COVID-19 (AĞALAR; ENGIN; ÖZTÜRK, 2020; CARVALHO et al., 2020).

A Associação Brasileira de Medicina de Emergência (ABRAMEDE) corroborou com uma publicação em maio, com recomendações quanto à paramentação, desparamentação e reutilização cíclica de equipamentos. A paramentação adequada deve seguir a seguinte sequência: (1) antissepsia das mãos (podendo ser feita com álcool $70 \%$ ou água e sabão); (2) vestimenta da máscara preconizada para a situação de atendimento; (3) nova antissepsia das mãos é feita para que seja feita a (4) colocação do óculos de proteção; (5) antissepsia das mãos é realizada mais uma vez para que seja feita a (6) vestimenta do propé; (7) nova antissepsia das mãos é feita para (8) vestimenta do macacão e do gorro; (9) mais uma antissepsia é feita para que a (10) viseira de proteção (face shield) seja colocada, se necessário; (11) nova antissepsia é feita para que seja realizada a (12) vestimenta de luvas, (13) do avental impermeável e (14) vestimenta de um segundo par de luvas (AMOROSO et al., 2020).

\section{Modo correto de desparamentação}

Amoroso et al., (2020) ainda contribui afirmando que a desparamentação feita de forma adequada é tão importante quanto a paramentação para diminuir as chances de infecção pela COVID-19.

A desparamentação adequada deve seguir a seguinte sequência: (1) retirada do avental impermeável pelas laterais, seguida do primeiro par de luvas; (2) antissepsia das mãos enluvadas com álcool a 70\%; (3) retirada da viseira "face shield" caso presente; (4) higienização da "face shield" com solução de álcool a 70\% ou soluções desinfetantes; (5) retirada do macacão iniciando pelo gorro; (6) retirada dos propés; (7) retirada do último par de luvas; (8) antissepsia das mãos com álcool a 70\%; (9) retirada dos óculos de proteção de posterior para anterior; (10) antissepsia das mãos com álcool a 70\%; (11) retirada das máscaras de posterior para anterior; (12) antissepsia das mãos com álcool a 70\%.

\section{Consequências do uso e desparamentação inadequados}

De acordo com o guia publicado pela OMS, em 5 de junho, para que os EPIs sejam efetivos, é preciso seguir as orientações adequadas de uso. Com relação às máscaras 


\section{RECIMA21 - REVISTA CIENTÍFICA MULTIDISCIPLINAR ISSN 2675-6218}

UTILIZAÇÃO DOS EQUIPAMENTOS DE PROTEC̃̃O INDIVIDUAL A PARTIR DA ÓTICA DA EQUIPE DE ENFERMAGEM NO ENFRENTAMENTO DO COVID-19 DA EQUIPE DE ENFERMAGEM NO ENFRENTAMENTO DO COVID-19
Júlio Gabriel Mendonça de Sousa, Wanderson Alves Ribeiro, Amanda de Araujo Franco, Amanda de Almeida Floriano, Ana Beatriz Teodoro de Souza, Bianca Lemos de Carvalho, Jully Camara Guinancio, Lilian Laine da Conceição Dias

cirúrgicas, quando utilizada apenas uma durante o plantão, é preciso garantir sua integridade, checar se há sujidade visível ou umidade. Ao ajustá-la à face, deve-se remover de forma segura pelas laterais, não podendo tocar a parte anterior da mesma e o descarte feito corretamente com a higiene das mãos realizada na sequência (OMS, 2020; CARVALHO et al., 2020).

A OMS (2020) e CARVALHO et al., (2020) ainda complementam que os riscos relacionados ao mau uso dos EPls são a auto contaminação pela manipulação errada das máscaras com as mãos contaminadas e/ou a falsa sensação de segurança que diminui a aderência às medidas preventivas complementares. O uso seletivo de materiais também é inadequado. Quando usada isoladamente por profissionais de saúde, as máscaras não blindam todas as possíveis vias de contaminação, como seria o caso da proteção ocular associada.

OMS (2020) e CARVALHO et al., (2020) ainda corroboram que, além disso, a proteção ocular junto da máscara reduz a possibilidade de ao tocar a máscara, levar as mãos aos olhos por acidente. Como as máscaras são a forma mais acessível para o público geral, muito se especifica sobre sua manutenção.

Diante do exposto, cabe ressaltar que as recomendações incluem 0 não compartilhamento das máscaras entre pessoas; quando molhadas, que sejam feitos os descartes adequados e as trocas após higiene das mãos. Não há especificação relevante com relação ao tempo de uso, mas existe sobre a composição do material utilizado e suas condições durante o uso (OMS, 2020; CARVALHO et al., 2020).

\section{Lesões de pele por utilização de EPIs}

Uma das consequências do uso dos EPIs por tempo prolongado e contínuo são as lesões de pele, sendo que a prevalência dos danos cutâneos pode chegar até $97 \%$ entre os profissionais de saúde da linha de frente. A pele é a primeira linha de defesa contra o meio ambiente, por isso é fundamental manter essa barreira íntegra para evitar contaminações (ELSTON, 2020; ALVES et al., 2020; CARVALHO et al., 2020).

Vale informar que a utilização inadequada e contínua dos EPIs implica forças mecânicas e químicas sobre a pele, agredindo-a e alterando suas características protetoras. Fatores físicos, como pressão sustentada, forças de tensão, fricção e atrito, bem como a umidade e a temperatura, estão diretamente associadas ao desenvolvimento de úlceras e lesões por fricção, principalmente nas regiões de ponte nasal, mãos, bochecha e testa. Estas lesões podem aumentar o risco de infecção, causar dor e cicatrizes, resultando numa qualidade de vida reduzida (ELSTON, 2020; ALVES et al., 2020; CARVALHO et al., 2020).

Nesse sentido, outro fator que pode afetar a saúde do profissional é a higienização constante e o uso de luvas, que aumentam o risco de dermatites e dermatoses, podendo causar 


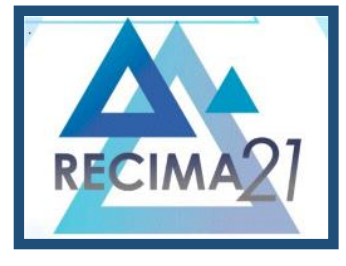

\section{RECIMA21 - REVISTA CIENTÍFICA MULTIDISCIPLINAR ISSN 2675-6218}

UTILIZAÇÃO DOS EQUIPAMENTOS DE PROTEC̃̃O INDIVIDUAL A PARTIR DA ÓTICA DA EQUIPE DE ENFERMAGEM NO ENFRENTAMENTO DO COVID-19 DA EQUIPE DE ENFERMAGEM NO ENFRENTAMENTO DO COVID-19
Júlio Gabriel Mendonça de Sousa, Wanderson Alves Ribeiro, Amanda de Araujo Franco, Amanda de Almeida Floriano, Ana Beatriz Teodoro de Souza, Bianca Lemos de Carvalho, Jully Camara Guinancio, Lilian Laine da Conceição Dias

eritema, ressecamento, descamação, fissuras, prurido, infecções secundárias e agravamento de doenças de pele (ELSTON, 2020; ALVES et al., 2020; CARVALHO et al., 2020).

Para evitar as complicações dos EPls relacionadas à pele são recomendados: (1) cuidados com a pele antes e após a utilização dos EPIs, utilizando cremes hidratantes, protetores de barreira; (2) utilização de material de interface (como bandagem ou esparadrapo) entre o EPI e a pele nas zonas de adesão/pressão/fricção; (3) realizar a lavagem das mãos associado ao uso de substâncias cosméticas que podem ajudar no processo de retenção e redução da perda de água transepidérmica; (4) programar minutos de alívio de pressão das EPIs, planejando turnos de rotação mais curtos em equipamentos de alta intensidade protetora, sempre tomando cuidado com a contaminação; (5) tratar, proteger e evitar o uso de máscara e óculos de proteção sobre áreas afetadas (ELSTON, 2020; ALVES et al., 2020; CARVALHO et al., 2020).

\section{CONSIDERAÇÕES FINAIS}

A pandemia trouxe tempos inigualáveis, instáveis e com a velocidade das mudanças rápidas de forma crescente, no entanto, informações confiáveis, atuais e de boa qualidade não são suficientes, faz-se necessário também pensar no papel da comunicação de forma adequada e eficaz de eliminar os riscos, ressaltar a importância da higienização das mãos e uso contínuo da máscara de forma correta. Nesse momento, faz-se necessário utilizar os canais de mídias de forma contínua.

Por sua vez, o envolvimento de toda sociedade para a adoção consciente das medidas de precaução frente à COVID-19 exige uma mudança de comportamento individual e coletivo nesse momento, de forma imediata e rigorosa. Nesse cenário da pandemia, é possível aprender que seu curso e impactos no país depende do esforço colaborativo de todos, poder público, profissionais de saúde e cidadãos.

Por fim, a enfermagem constitui a maior representatividade de pessoal dentro do hospital. No desempenho de suas atividades impõem-se rotinas, elevadas cargas horárias semanais, número reduzido de pessoal para cumprir suas funções, contato com substâncias, compostos ou produtos químicos em geral. Além do risco biológico permanente, somam-se ainda esforço físico, levantamento e transporte manual de peso, materiais inadequados ou defeituosos, postura corporal inadequada, trabalho noturno, iluminação deficiente e situações causadoras de estresse psíquico.

\section{REFERÊNCIAS}

AĞALAR, Canan; ENGIN, Derya Öztürk. Protective measures for COVID-19 for healthcare providers and laboratory personnel. Turkish journal of medical sciences, v. 50, n. SI-1, p. 578$584,2020$. 


\section{RECIMA21 - REVISTA CIENTÍFICA MULTIDISCIPLINAR ISSN 2675-6218}

UTILIZAÇÃO DOS EQUIPAMENTOS DE PROTEÇÃO INDIVIDUAL A PARTIR DA ÓTICA DA EQUIPE DE ENFERMAGEM NO ENFRENTAMENTO DO COVID-19 DA EQUIPE DE ENFERMAGEM NO ENFRENTAMENTO DO COVID-19
Júlio Gabriel Mendonça de Sousa, Wanderson Alves Ribeiro, Amanda de Araujo Franco, Amanda de Almeida Floriano, Ana Beatriz Teodoro de Souza, Bianca Lemos de Carvalho, Jully Camara Guinancio, Lilian Laine da Conceição Dias

ALMEIDA, Ildeberto M. Proteção da saúde dos trabalhadores da saúde em tempos de pandemia e respostas à pandemia. Rev. bras. saude ocup, São Paulo, v. 45, jun. 2020.

ALVES, P. et al. COVID19. Prevenção de lesões cutáneas causadas pelos Equipamentos de Proteção Individual (Máscaras faciais, respiradores, viseiras e óculos de proteção). Journal of Tissue Healing and Regeneration, 2020.

AMOROSO, D. et al. Recomendações para Reutilização Cíclica Racional de Equipamentos de Proteção Individual Durante a Pandemia por Covid-2019. Porto Alegre: ABRAMEDE, 2020.

ANDERSON; R. M; HEESTERBEEK, H; KLINKENBERG, D; HOLLINGSWORTH, T. D. How will country-based mitigation measures influence the course of the COVID-19 epidemic? Lancet, $v$. 395 , n. 10228, p. 931-4, 2020.

BÁO, A. C. P. .; AMESTOY, S. C. .; BERTOlDI, K.; BARRETO, L. N. M. .; NOMURA, A. T. G. .; SILVEIRA, J. C. Patient safety in the face of the COVID-19 pandemic: theoretical-reflective essay. Research, Society and Development, [S. I.], v. 9, n. 11, p. e73091110252, 2020. DOI: 10.33448/rsd-v9i11.10252.

BARROS, J. S.O.; RODRIGUES, A. P.; MIRANDA, L. N.; ARAÚJO, M. A. S. A enfermagem e a resistência ao uso dos equipamentos de proteção individual. Cad Graduação, v. 3, n. 3, p.189, 2016.

BRASIL. Legislativo. Lei n o 6.514, de 22 de dezembro de 1977. Altera o Capítulo V do Título II da Consolidação das Leis do Trabalho, relativo a segurança e medicina do trabalho e dá outras providências.

BRASIL. Resumo da Nota Técnica da ANVISA: medidas de prevenção que devem ser adotadas na assistência a pacientes com suspeita ou confirmação de covid-19". "Medidas de Prevenção que devem ser adotadas na assistência a pacientes com suspeita ou confirmação de COVID-19". [S.I.]: Sociedade Brasileira de Infectologia, 2020.

BRUM, C. N.; ZUGE, S. S.; RANGEL, R. F.; FREITAS, H. M. B.; PIESZAK, G. M. Revisão narrativa da literatura: aspectos conceituais e metodológicos na construção do conhecimento da enfermagem. In.: LACERDA, M. R.; COSTENARO, R. G. S. Metodologias da pesquisa para a enfermagem e saúde. Porto Alegre: Moriá, 2015.

CHANG, L. E.; YAN, Y.; WANG, L. Coronavirus disease 2019: Coronaviruses and blood safety. TransfusMed Rev, Feb, 2020.

COFEN, Conselho Federal de Enfermagem. Nota técnica $n^{0}$ 01/2020. Orientações sobre o novo coronavírus (Covid-19). Enferm. Foco, Brasília, v. 8-9, 2020.

COOK, T. M. Personal protective equipment during the coronavirus disease (COVID) 2019 pandemic-a narrative review. Anaesthesia, v. 75, n. 7, p. 920-927, 2020.

CARVALHO, Raphael Salomão et al. Utilização de equipamentos de proteção individual em época de COVID-19. Global Academic Nursing Journal, v. 1, n. 1, p. e6-e6, 2020.

DIAS, J. Á.; SILVA, P. E.; ALVES, S. R. P.; SOUTO, C. G. V. Nursing students of knowledge about the equipment personal protection (PPE) used in emergency unit and emergency hospital. RevCiênc Saúde Nova Esperança, v. 14, n. 1, p. 4-14, 2016. 


\section{RECIMA21 - REVISTA CIENTÍFICA MULTIDISCIPLINAR ISSN 2675-6218}

UTILIZAÇÃO DOS EQUIPAMENTOS DE PROTEÇÃO INDIVIDUAL A PARTIR DA ÓTICA DA EQUIPE DE ENFERMAGEM NO ENFRENTAMENTO DO COVID-19 DA EQUIPE DE ENFERMAGEM NO ENFRENTAMENTO DO COVID-19
Júlio Gabriel Mendonça de Sousa, Wanderson Alves Ribeiro, Amanda de Araujo Franco, Amanda de Almeida Floriano, Ana Beatriz Teodoro de Souza, Bianca Lemos de Carvalho, Jully Camara Guinancio, Lilian Laine da Conceição Dias

ELSTON, Dirk M. Doença de pele ocupacional em trabalhadores da saúde durante a epidemia de coronavírus (COVID-19). Jornal da Academia Americana de Dermatologia, v. 82, n. 5, p. 1085, 2020.

FAUCI, A. S.; LANE, H. C.; REDFIELD, R. R. Covid-19 - Navigating the Uncharted. N Engl J Med, v. 382, e1268-9, 2020.

GARCIA, L. P. Uso de máscara facial para limitar a transmissão da COVID-19. EpidemioIServ Saúde. No prelo. 2020.

GIL, A. C. Métodos e técnicas de pesquisa social. 6. ed. São Paulo: Atlas, 2008.

RIBEIRO JUNIOR, M. A. F. et al. O cirurgião de trauma e emergência na era da pandemia de COVID-19. Revista do Colégio Brasileiro de Cirurgiões, v. 47, 2020.

LUZ, Alessandra Rocha et al. COVID-19: medidas de prevenção de lesão por pressão ocasionadas por equipamentos de proteção individual em profissionais da saúde. Revista Enfermagem Atual In Derme, v. 93, 2020.

MARZIALE, M. H. P.; GALON, T.; SOUZA, W. L. A legislação brasileira e as recomendações internacionais sobre a exposição ocupacional aos agentes. Rev. brasileira Enfermagem, Brasília, v. 64, n. $1,2011$.

OLIVEIRA, A. C.; LUCAS, T. C.; IQUIAPAZA, R. A. O que a pandemia da Covid-19 tem nos ensinado sobre adoção de medidas de precaução?.TextoContextoEnferm, v. 29, e20200106, 2020.

OLIVEIRA, Kauan Tamandaré et al. Principais medidas tomadas para a mudança dos processos assistenciais durante a pandemia por COVID-19. Enferm. Foco, Brasília, p. 235-238, 2020.

OMS. Tipos recomendados de equipamentos de proteção individual no contexto do COVID19, de acordo com o tipo de ambiente, pessoa alvo e tipo de atividade. [S. I.]: Organização Mundial da Saúde, 2020.

SANT'ANA, Geisa et al. Infecção e óbitos de profissionais da saúde por COVID-19: revisão sistemática. Acta Paulista de Enfermagem, v. 33, 2020.

SARAIVA, Emanuela Machado Silva et al. Impacto da pandemia pelo Covid-19 na provisão de equipamentos de proteção individual. Brazilian Journal of Development, v. 6, n. 7, p. 4375143762, 2020.

SOARES, Samira Silva Santos et al. Pandemia de Covid-19 e o uso racional de equipamentos de proteção individual. Covid-19 pandemic and rational use of personal protective equipment Pandemia de Covid-19 y uso racional de equipos de protección personal]. Revista enfermagem uerj, v. 28, p. 50360, 2020.

SUARTE, H. A.; TEIXEIRA, P. L.; RIBEIRO, M. S. O uso dos equipamentos de proteção individual e a prática da equipe de enfermagem no centro cirúrgico. RevCient do ITPAC, v. 6, n. 2, p.1-8, 2013.

TALHAFERRO, B.; BARBOZA, D. B.; OLIVEIRA, A. R. Adesão ao uso dos equipamentos de proteção individual pela enfermagem. Rev CiêncMéd. v. 17, n. 3-6, p.157-66, 2008. 


\section{RECIMA21 - REVISTA CIENTÍFICA MULTIDISCIPLINAR ISSN 2675-6218}

UTILIZAÇÃO DOS EQUIPAMENTOS DE PROTEĈ̃O INDIVIDUAL A PARTIR DA ÓTICA DA EQUIPE DE ENFERMAGEM NO ENFRENTAMENTO DO COVID-19 Júlio Gabriel Mendonça de Sousa, Wanderson Alves Ribeiro, Amanda de Araujo Franco, Amanda de Almeida Floriano, Ana Beatriz Teodoro de Souza, Bianca Lemos de Carvalho, Jully Camara Guinancio, Lilian Laine da Conceição Dias

WALKER, P. G. T.; WHITTAKER, C.; WATSON, O. et al. The Global Impact of COVID-19 and Strategies for Mitigation and Suppression. Londres: Imperial College London, 2020.

WEISSMANN L, et al. Nota de Esclarecimento da Sociedade Brasileira de Infectologia: uso de máscaras na pandemia de COVID-19. São Paulo: [S. e.], 2020.

WHO. Advice on the use of mascks in the context of COVID-19. World Health Organization, 2020.

WORD HEALTH ORGANIZATION. Considerations for quarantine of individuals in the context of containment for coronavirus disease (COVID-19): Interim guidance. 